\title{
EFFECT OF OBESITY AND DIABETES MELLITUS TYPE 2 ON VASCULAR STIFFNESS
}

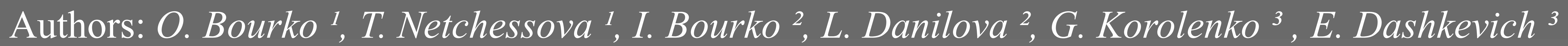

Hospital: 1 - Republican Scientific and Practical Center Cardiology, Minsk Belarus;

2 - Belarusian Medical Academy of Postgraduate Education, Minsk Belarus; 3 - 10 City Hospital, Minsk Belarus

\section{OBJECTIVES}

To investigate the impact of overall obesity $(\mathrm{Ob})$ and diabetes mellitus type 2 (DM2) on arterial stiffness and degree of insulin resistance in patients with arterial hypertension (AH) and abdominal obesity (AOb).

\section{METHODS}

74 subjects were divided according to presence of $\mathrm{AH}, \mathrm{AOb}, \mathrm{Ob}$ and $\mathrm{DM} 2$ without insulin therapy. Control group 1 consisted of 26 subjects without $\mathrm{AH}$, $\mathrm{AOb}, \mathrm{Ob}$ and $\mathrm{DM} 2$. All patients with $\mathrm{AH}$ were also diagnosed with $\mathrm{AOb}$ and amounted to 48 subjects. Then patients with $\mathrm{AH}$ and $\mathrm{AOb}$ were divided into two groups (group 2 and 3 respectively) according to presence of $\mathrm{Ob}$, defined by BMI and furthermore, DM2. Carotid-femoral pulse wave velocity (PWVcf) measurements were performed using SphygmoCor. Homeostasis model assessment-insulin resistance (HOMA-IR) was calculated by the following formula: fasting plasma insulin $(\mathrm{mU} / \mathrm{ml}) \quad \mathrm{x}$ fasting plasma glucose $(\mathrm{mmol} / \mathrm{l}) / 22.5$.

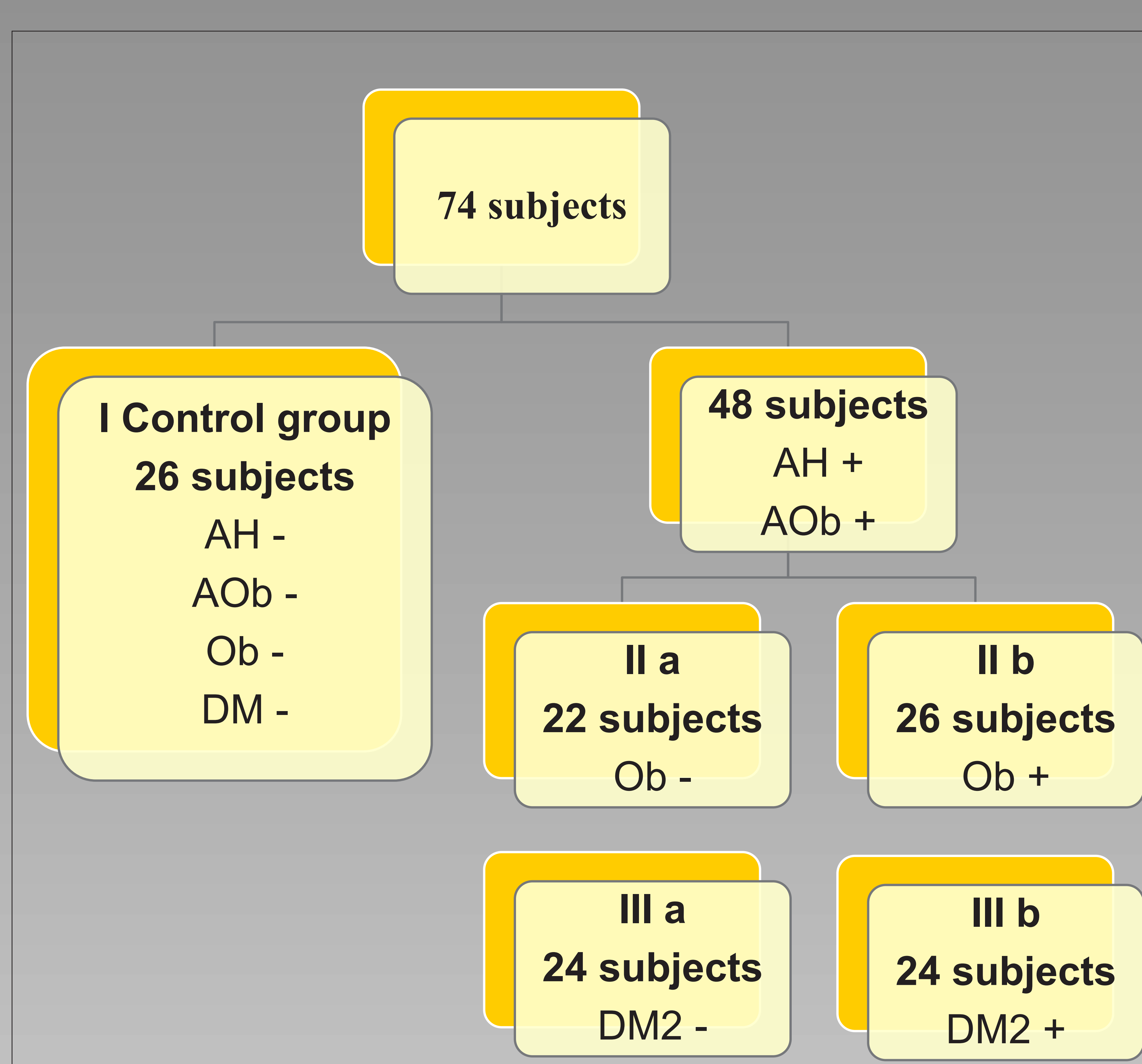

\section{CONCLUSIONS}

Conclusions Presence of overall $\mathrm{Ob}$ together with $\mathrm{AOb}$ had a significant adverse effect on arterial stiffness in patients with AH both men and women. This adverse effect is similar with impact of DM2 on arterial stiffness in patients with AH together with AOb. Measurement of PWVc-f showed higher significance versus HOMA-IR measurements in the study groups.

\section{RESULTS}

Results In the group comparison by BMI: PWVc-f and HOMA-IR increased consistently from Group 1 to Group 3. PWVc-f was significantly higher in hypertensive patients with $\mathrm{AOb}$ and $\mathrm{Ob}$ than in hypertensive patients with $\mathrm{AOb}$ and without $\mathrm{Ob}$ (PWVc$\mathrm{f}=8,69 \pm 1,8 ; \mathrm{PWV} c-\mathrm{f}=7.43 \pm 1,3 ; \mathrm{p}<0.05)$. HOMA-IR did not show significance. In the group comparison by presence of DM2: PWVc-f and HOMA-IR increased consistently from Group 1 to Group 3. PWVc-f and HOMA-IR were significantly higher in hypertensive patients with $\mathrm{AOb}$ and $\mathrm{DM} 2$ than in hypertensive patients with Aob and without DM2 (PWVc-f $=9.5 \pm 1.8$ and PWVc$\mathrm{f}=7.71 \pm 1.5, \quad \mathrm{p}<0.001$; and HOMA-IR $=7.09 \pm 3.54$ and HOMA$\mathrm{IR}=2.83 \pm 1.2 ; \mathrm{p}<0.001)$. Significant differences between groups persisted after adjustment for age, sex and BMI.

\section{REFERENCES:}

1. Hypertension Primer: The Essentials of High Blood Pressure: Basic Science, Population Science, and Clinical Management 2007 By Joseph L Izzo (Editor), Domenic Sica (Editor), Henry R Black (Editor) 2. Laurent S, Boutouyrie P, Asmar R, Gautier I, Laloux B, Guize L, Ducimetiere P, Benetos A:Aortic stiffness is an independent predictor of allcause and cardiovascular mortality in hypertensive patients.

Hypertension 2001, 37(5):1236-1241.

3. Vlachopoulos C, Aznaouridis K, Stefanadis C: Prediction of cardiovascular events and all-cause mortality with arterial stiffness: a systematic review and meta-analysis. J Am Coll Cardiol 2010, 55(13):1318-1327. 